\title{
Infiltration of T Cells and Programmed Cell Death Ligand 1-expressing Macrophages as a Potential Predictor of Lenvatinib Response in Hepato- cellular Carcinoma
}

\author{
Pil Soo Sung ${ }^{1,2}$, Sung Woo Cho', Jaejun Lee ${ }^{1,3}$, Hyun Yang ${ }^{1,3}$, Jeong Won Jang ${ }^{1,2}$, Si Hyun Bae ${ }^{1,3}$, Jong Young Choi ${ }^{1,2}$, \\ Seung Kew Yoon ${ }^{1,3}$ \\ ${ }^{1}$ The Catholic University Liver Research Center, College of Medicine, The Catholic University of Korea, Seoul; ${ }^{2}$ Division of Gastroenter- \\ ology and Hepatology, Department of Internal Medicine, Seoul St. Mary's Hospital, College of Medicine, The Catholic University of \\ Korea, Seoul; ${ }^{3}$ Division of Gastroenterology and Hepatology, Department of Internal Medicine, Eunpyeong St. Mary's Hospital, College \\ of Medicine, The Catholic University of Korea, Seoul, Korea
}

Received Jun. 13, 2020

Revised Jul. 27, 2020

Accepted Jul. 30, 2020
Background/Aims: Lenvatinib was recently proven to be non-inferior to sorafenib in treating unresectable hepatocellular carcinoma (HCC) in a phase-3 randomized controlled trial. In this study, we investigated whether the response to lenvatinib was affected by tumor immunogenicity.

Methods: Between May 2019 and April 2020, nine patients with intermediate-to-advanced HCC, who were treated with lenvatinib after liver biopsy, were enrolled. Immunohistochemical staining and multi-color flow cytometry were performed on specimens obtained from liver biopsy.

Results: Among the nine patients enrolled, four showed objective responses (complete responses+partial responses). Immunohistochemical staining for $\mathrm{CD} 3, \mathrm{CD} 68$, and programmed cell death ligand 1 (PD-L1) demonstrated that patients with objective responses showed marked infiltration of T cells and PD-L1-expressing macrophages in intra-tumoral and peri-tumoral tissues compared to those without objective responses. A significant difference in the numbers of infiltrated T cells, both in the intra-tumoral $(P<0.01)$ and peri-tumoral regions $(P<0.05)$, were identified between responders and non-responders. Regarding the number of infiltrated macrophages, no significant difference was found between the responders and non-responders, although the number of PD-L1-expressing tumor-associated macrophages was significantly higher in responders than that in non-responders $(P<0.05)$.

Conclusions: Tumor immunogenicity, as indicated by T cell and PD-L1-positive macrophage infiltration, affects lenvatinib response in unresectable HCC. (J Liver Cancer 2020;20:128-134)

Keywords: Hepatocellular carcinoma; Lenvatinib; PD-L1; Tumor-associated macrophage; Objective response

\section{Corresponding author: Pil Soo Sung}

Division of Gastroenterology and Hepatology, Department of Internal Medicine, College of Medicine, The Catholic University of Korea, 222 Banpo-daero, Seocho-gu, Seoul 06591, Korea

Tel. +82-2258-2073, Fax. +82-2-3481-4025

E-mail; pssung@catholic.ac.kr

https://orcid.org/0000-0002-5780-9607

\section{INTRODUCTION}

Liver cancer is the fourth most common cause of malignancy-associated mortality. ${ }^{1}$ Hepatocellular carcinoma (HCC) accounts for $80 \%$ of the primary malignant liver cancers. ${ }^{1}$ In 2007, sorafenib, a multi-target tyrosine kinase inhibitor, was approved for the treatment of unresectable HCC. 
Although sorafenib improves the overall survival of patients with unresectable HCC, the survival benefit is modest. ${ }^{2,3}$ Similar to sorafenib, lenvatinib is an oral multi-target tyrosine kinase inhibitor. Evidence from preclinical studies indicates that its potent antiangiogenic activity is associated with the inhibition of the fibroblast and vascular endothelial growth factor (VEGF) signaling pathways. ${ }^{4}$ A recent international multi-center clinical trial comparing lenvatinib with sorafenib as the first-line treatment for unresectable HCC found that the median overall survival of patients treated with lenvantinib was non-inferior to that of patients treated with sorafenib, although lenvatinib was superior to sorafenib in terms of objective response rate and progression-free survival. ${ }^{5}$ Consequently, lenvatinib was approved as the first-line therapy for unresectable HCC in countries including Korea, Japan, China, the EU, and the USA. ${ }^{6,7}$

HCC is recognized as an immune-tolerant malignancy. ${ }^{8}$ Overexpression of immune inhibitory molecules, such as programmed cell death protein-1 (PD-1), leads to the exhaustion of activated CD8+ T cells. ${ }^{1,9}$ Moreover, the infiltration of various immune cells, such as tumor-associated macrophages (TAMs), leads to an immunosuppressive microenvironment. ${ }^{10,11}$ However, only $14-18 \%$ of the patients who receive pembrolizumab or nivolumab monotherapy demonstrate objective tumor responses. ${ }^{12-14}$ Moreover, unlike that in other solid tumors, no significant association was identified between tumor cell programmed cell death ligand 1 (PD-L1) expression and anti-PD-1 responses in HCC, as reported by the Keynote-224 and CheckMate-040 studies. $^{9,15,16}$

A previous report has demonstrated that lenvatinib requires the help of CD8+ T cells to exert antitumor activity. ${ }^{17}$ Moreover, tumor volume was significantly reduced through the use of lenvatinib in combination with the anti-PD-1 antibody in a mouse syngeneic Hepa1-6 HCC tumor model. ${ }^{17}$ These findings in mice have suggested that lenvatinib exerts immunomodulatory and antitumor effects. In this study, we evaluated the role of infiltrating immune cells in lenvatinibtreated unresectable HCC through the application of immunohistochemistry and multicolor flow cytometry of patient's samples, which were obtained by biopsy.

\section{METHODS}

This study was conducted after approval from the Institutional Review Boards of Seoul St. Mary's Hospital and Eunpyeong St. Mary's Hospital (XC20RIDI0063).

\section{Study design and population}

This study was approved by the Institutional Review Board of Eunpyeong St. Mary's Hospital and Seoul St. Mary's Hospital (XC20RIDI0063). We retrospectively reviewed the medical records of nine patients with unresectable HCC who underwent liver biopsy prior to lenvatinib treatment at these hospitals between May 2019 and April 2020. The most recent guidelines issued by the European Association for the Study of the Liver and the American Association for the Study of Liver Diseases were used to diagnose HCC. ${ }^{18,19}$ The diagnostic methods included imaging with multiphasic computed tomography and magnetic resonance imaging and serum assays for alpha-fetoprotein (AFP) and other tumor markers. Liver biopsies were performed for assessment via immunohistochemistry and flow cytometry.

\section{Response evaluation}

Lenvatinib treatment response was evaluated 6-8 weeks after treatment initiation using the modified RECIST criteria. ${ }^{20}$ In cases where none of the target lesions demonstrated arterial enhancement, the tumor was categorized as being in complete remission. Where the sum of the diameters of viable target lesions was reduced by $30 \%$, remission was defined as partial (PR). In contrast, when the sum of viable target lesions increased by $20 \%$ or more, the disease was categorized as progressive (PD). Any diseased state that did not meet the criteria of PR or PD was defined as stable., ${ }^{3,20}$

\section{Flow cytometry}

The following antibodies were used for multi-color flow cytometry: BV510-conjugated anti-CD163, APC-H7-conjugated anti-Human Leukocyte Antigen-DR isotype (HLA- 
DR) (BD Biosciences, San Jose, CA, USA), PE-eFluor 610-conjugated anti-CD14 (eBioscience Inc., San Diego, CA, USA), PE/Cy7-conjugated anti-PD-L1, and APC-conjugated anti-CD206 (BioLegend, San Diego, CA, USA). Multi-color flow cytometry was performed using the LSR Fortessa (Becton Dickinson, Franklin Lakes, NJ, USA ), and data were analyzed using the FlowJo software (TreeStar, Ashland, OR, USA) as per previously described methods. ${ }^{21}$

\section{Statistical analyses}

The SPSS version 26 software (IBM Corp., Armonk, NY, USA) was used for data analysis. The categorical variables associated with two groups (i.e., patients demonstrating responses to lenvatinib treatment and those not presenting responses) were analyzed using a chi-square test, and the groups' continuous variables were assessed using an independent $t$-test. Results with $P<0.05$ were regarded as statistically significant.

\section{RESULTS}

\section{Patient characteristics}

The baseline clinical characteristics of the nine enrolled patients are shown in Table 1. The median patient age was 57 years, and the principal etiology of HCC was hepatitis B virus infection (67\%). Extrahepatic metastases were reported in one patient, and portal vein tumor thrombosis was identified in four patients. The median alpha-fetoprotein (AFP) level was $2,031 \mathrm{ng} / \mathrm{mL}$. All patients were classified as having ChildPugh class A at the time of lenvatinib start. Prior to systemic therapy, most patients underwent local-regional therapies, such as trans-arterial chemoembolization or hepatic arterial infusion chemotherapy. The median lenvatinib treatment duration was 9 weeks. Patients with bodyweights of more than $60 \mathrm{~kg}$ received $12 \mathrm{mg}$ of lenvatinib and those weighing less than $60 \mathrm{~kg}$ received $8 \mathrm{mg}$.
Table 1. Baseline characteristics of the enrolled patients

\begin{tabular}{|c|c|}
\hline Clinical parameter & Value $(n=9)$ \\
\hline Median age (range, years) & $57(43-76)$ \\
\hline Sex (male) & $9(100)$ \\
\hline HBsAg-positive & $6(67)$ \\
\hline Anti-HCV-positive & $1(11)$ \\
\hline Median tumor size (cm) & 6.5 \\
\hline$<5 \mathrm{~cm}$ & $3(33)$ \\
\hline$\geq 5 \mathrm{~cm}$ & $6(67)$ \\
\hline Multiple tumors & $7(78)$ \\
\hline Portal vein tumor thrombosis & $4(44)$ \\
\hline Extrahepatic metastasis & $1(11)$ \\
\hline Median AFP (range, ng/mL) & 2,031 (2.1-160,000) \\
\hline \multicolumn{2}{|l|}{ Child-Pugh score } \\
\hline 5 & $5(56)$ \\
\hline 6 & $4(44)$ \\
\hline \multicolumn{2}{|l|}{ Prior therapy to lenvatinib } \\
\hline Surgical resection & $1(11)$ \\
\hline TACE/HAIC & $5(56)$ \\
\hline Sorafenib & 0 \\
\hline \multicolumn{2}{|l|}{ Best responses to nivolumab } \\
\hline Complete response & 0 \\
\hline Partial response & $4(44)$ \\
\hline Stable disease & $2(22)$ \\
\hline Progressive disease & $3(33)$ \\
\hline
\end{tabular}

Values are presented as number (\%) unless otherwise indicated. $\mathrm{HBsAg}$, hepatitis B surface antigen; HCV, hepatitis C virus; AFP, alpha fetoprotein; TACE, transarterial chemoembolization; HAIC, hepatic arterial infusion chemotherapy.

\section{Objective responses to lenvatinib were associ- ated with intra-tumoral/peri-tumoral infiltration of $\mathrm{T}$ cells or PD-L1-expressing macrophages}

First, we compared the frequency of intra-tumoral or peritumoral $\mathrm{T}$ cells and macrophages between lenvatinib responders $(\mathrm{CR}+\mathrm{PR}, \mathrm{n}=4)$ and non-responders $(\mathrm{SD}+\mathrm{PD}, \mathrm{n}=5)$ using immunohistochemistry data (Fig. 1). Lenvatinib responders showed a significantly higher frequency of intra-tumoral (Fig. 1A) and peri-tumoral (Fig. 1B) T cells than lenvatinib non-responders. However, there were no significant differences in the frequency of intra-tumoral (Fig. 1C) and 
(A)

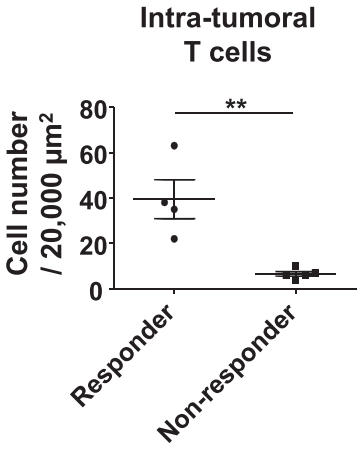

Intra-tumoral macrophages

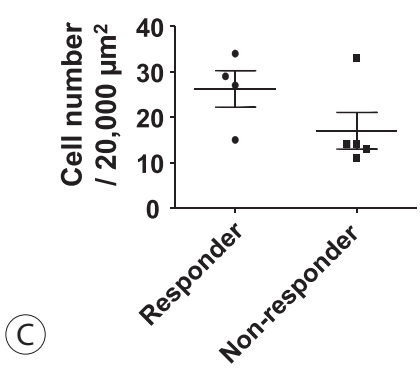

(B)

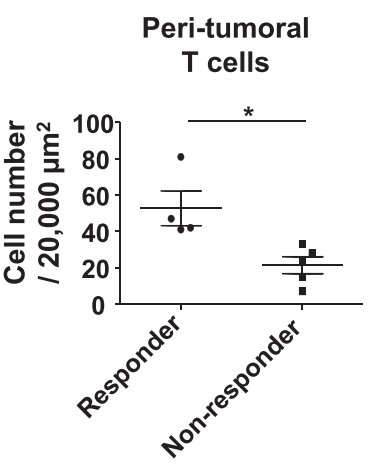

Peri-tumoral macrophages

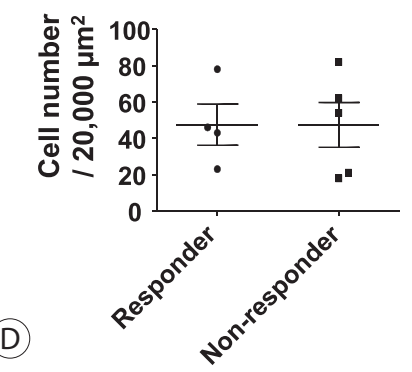

PD-L1+ cells

(E)

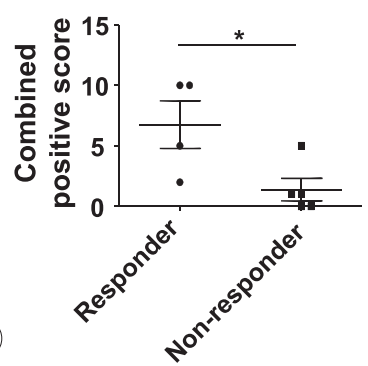

Figure 1. Objective responses to lenvatinib are associated with intra-tumoral/peri-tumoral infiltration of $\mathrm{T}$ cells or programmed cell death ligand 1 (PD-L1)-expressing cells. (A) Frequency of intra-tumoral T cells in lenvatinib responders $(n=4)$ and lenvatinib non-responders ( $n=5)$. (B) Frequency of peri-tumoral T cells in lenvatinib responders and lenvatinib non-responders. (C) Frequency of intra-tumoral macrophages in lenvatinib responders and lenvatinib non-responders. (D) Frequency of peri-tumoral macrophages in lenvatinib responders and lenvatinib non-responders. (E) Frequency of PD-L1-expressing cells in lenvatinib responders and lenvatinib non-responders. Bar graphs represent the mean \pm standard deviation. ${ }^{*} P<0.05$; ${ }^{\dagger} P<0.01$.

peri-tumoral (Fig. 1D) macrophages between lenvatinib responders and non-responders. Total PD-L1-expressing cells were represented by combined positive scores, and lenvatinib responders presented significantly higher numbers than nonresponders.

\section{Tumor-associated macrophages were the primary cells expressing PD-L1 in HCC}

Next, we investigated which cell types expressed PD-L1 in the tissues of lenvatinib responders. Fig. 2A is representative of the immunohistochemistry of lenvatinib responders. This panel includes three distinct parts: $\mathrm{T}$ cell compartment $(\mathrm{CD} 3+)$, macrophage compartment (CD68+), and tumorcell compartment. Interestingly, PD-L1 was generally expressed only in the macrophage compartment and was rarely expressed in T cell or tumor-cell compartments (Fig. 2A).
Flow cytometry using intra-tumoral liver biopsy specimens demonstrated that TAMs, which expressed high levels of CD206 and CD163, also expressed high levels of PD-L1 in lenvatinib responders. Moreover, PD-L1 expression showed a positive correlation with HLA-DR expression, thereby indicating that these TAMs might activate cognate effector $\mathrm{T}$ cells after PD-1/PD-L1 blockade (Fig. 2B). In contrast, lenvatinib non-responders showed less $\mathrm{CD} 3+\mathrm{T}$ cell infiltration in both peri-tumoral and intra-tumoral regions. Moreover, CD68+ TAMs did not express PD-L1 in lenvatinib non-responders, although a considerable number of TAMs infiltrated (Fig. 2C).

\section{DISCUSSION}

Lenvatinib was recently reported to exert significant immunomodulatory activity in a Hepal-6 mouse HCC model. ${ }^{17}$ 


\section{Lenvatinib responder}

CD3

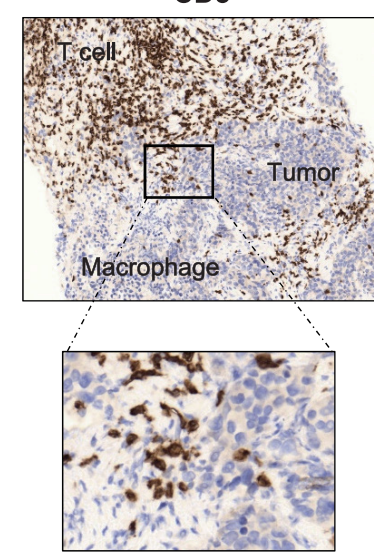

(A)

(B)

\section{Lenvatinib responder}

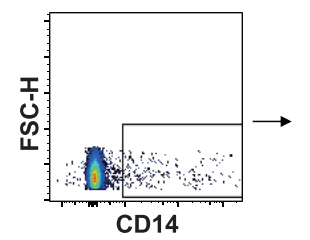

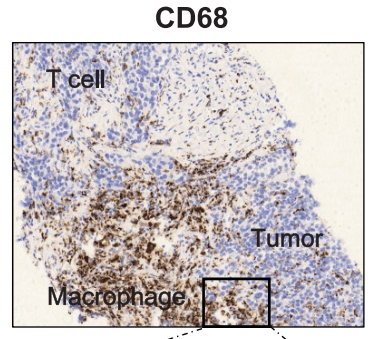

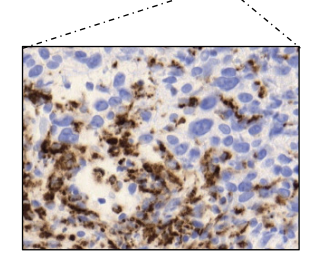

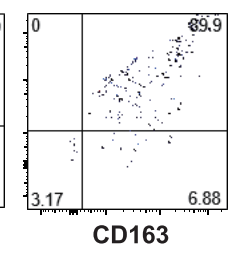

PD-L1
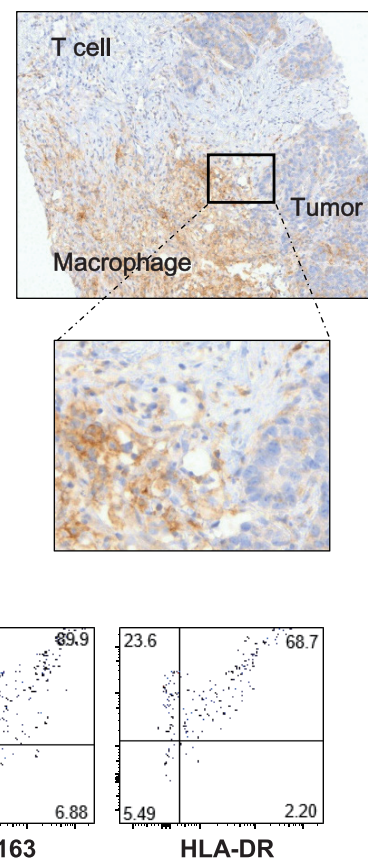

\section{Lenvatinib non-responder}
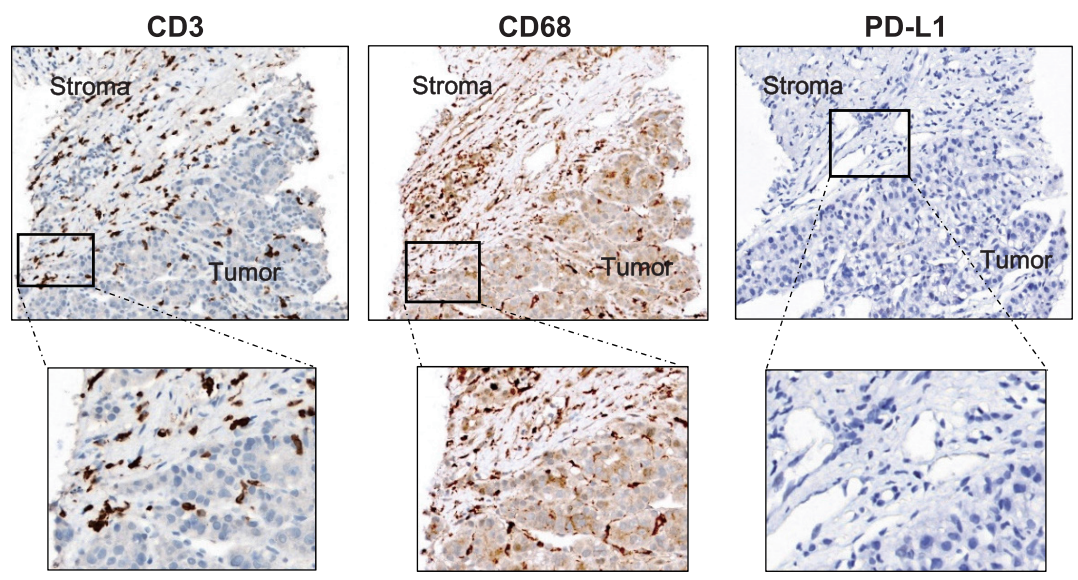

Figure 2. Tumor-associated macrophages are the primary cells expressing programmed cell death ligand 1 (PD-L1) in hepatocellular carcinoma after lenvatinib treatment. (A) Immunohistochemistry of CD3, CD68, and PD-L1 in the biopsy specimen of a lenvatinib responder. (B) Flow cytometry analysis of PD-L1, Human Leukocyte Antigen-DR isotype, CD206, and CD163 expression in intra-tumoral macrophages. (C) Immunohistochemistry of CD3, CD68, and PD-L1 in the biopsy specimen of a lenvatinib non-responder.

Another recent study using single-cell analysis has demonstrated that lenvatinib reduces the population of TAMs. ${ }^{22}$ These findings suggest that lenvatinib not only potently inhibits angiogenesis but also exerts considerable immunomodulatory effects. Moreover, recent reports have demon- strated that the expression of PD-1 and other inhibitory checkpoints is upregulated by VEGF-A secreted by cancer and stromal cells and that immune regulatory function is disrupted when VEGF receptor signaling is targeted with VEGF receptor inhibitors. ${ }^{23,24}$ These studies suggest that len- 
vatinib can promote antitumor immunity by inhibiting VEGF signaling pathways, resulting in the activation of $\mathrm{CD} 8+\mathrm{T}$ cells and a reduction in the TAM population.

Monocytic cells in the peripheral circulation are recruited to specific tumor microenvironments and become TAMs. ${ }^{25}$ In general, macrophages can be classified within two main groups: classically activated macrophages (M1) and alternatively activated macrophages (M2). ${ }^{25}$ In general, TAMs exhibit the M2 phenotype, and their infiltration is known to be associated with poor prognosis in HCC. ${ }^{9,11,26}$ However, the function and phenotypes of TAMs in human HCC tissues have not been precisely characterized thus far. In different cancer types, including lung cancer, conflicting results have been found regarding the correlation of TAM counts in surgical specimens and clinical prognoses. ${ }^{27}$ It appears that tumor-expressed PD-L1 directly inhibits T cells, but the significance of PD-L1 expression by TAMs for the regulation of the effector phase of tumor-specific T cells remains unclear. ${ }^{27}$ In HCC, PD-L1 expression is generally very weak in tumor cells, and the expression of PD-L1 in TAMs may justify tumor immunogenicity. Interestingly, TAMs in lenvatinib responders in our study seems not to be fully M2-polarized because they express high levels of HLA-DR, which is a crucial M1 marker. Therefore, it can be suggested that these tumors are immunogenic and can respond to treatments modulating antitumor immune responses.

Noteworthy, our study also suggests that treatment with lenvatinib plus anti-PD-1/PD-L1 blockade may be a promising combinatorial strategy for immune-based HCC therapy. In general, two different drugs with different mechanisms of $\mathrm{T}$ cell restoration have synergistic anti-tumor activity. Therefore, treatment with lenvatinib (anti-VEGF activity) + antiPD-1/PD-L1 will probably result in better responses in HCC than treatment only with the single agent.

This study includes the following limitations: 1) A retrospective study design, 2) a small number of patients, 3) a short follow-up period, 4) a single-arm design.

In conclusion, our study demonstrates that tumor immunogenicity, reflected by T cell and PD-L1-positive macrophage infiltration, affects the response of unresectable HCC to lenvatinib. Future studies with larger numbers of patients may elucidate the precise function of TAMs and the immune-modulatory function of lenvatinib treatment in HCC.

\section{ACKNOWLEDGMENTS}

This work was supported by the Scientific Research Fund of the Korean Liver Cancer Study Group. This work was supported by a National Research Foundation of Korea (NRF) grant funded by the Korean government (MSIT) (2020R1A2C3011569). English editing was performed by Editage (https://www.editage. co.kr).

\section{AUTHOR CONTRIBUTIONS}

Pil Soo Sung: study design, data collection, data analysis, data interpretation, manuscript writing, and manuscript approval.

Sung Woo Cho, Jaejun Lee, and Hyun Yang: data collection.

Jeong Won Jang, Si Hyun Bae, Jong Young Choi, and Seung Kew Yoon: data interpretation and manuscript approval.

\section{Conflicts of Interest}

The authors have no conflicts to disclose.

\section{REFERENCES}

1. Yoon SK. Molecular mechanism of hepatocellular carcinoma. Hepatoma Research 2018;4:42.

2. Dika IE, Abou-Alfa GK. Treatment options after sorafenib failure in patients with hepatocellular carcinoma. Clin Mol Hepatol 2017;23:273-279.

3. Sung PS, Park HL, Yang K, Hwang S, Song MJ, Jang JW, et al. (18) F-fluorodeoxyglucose uptake of hepatocellular carcinoma as a prognostic predictor in patients with sorafenib treatment. Eur J Nucl Med Mol Imaging 2018:45:384-391.

4. Bangaru S, Marrero JA, Singal AG. Review article: new therapeutic interventions for advanced hepatocellular carcinoma. Aliment Pharmacol Ther 2020;51:78-89.

5. Kudo M, Finn RS, Qin S, Han KH, Ikeda K, Piscaglia F, et al. Lenvatinib versus sorafenib in first-line treatment of patients with unresectable hepatocellular carcinoma: a randomised phase 3 noninferiority trial. The Lancet 2018;391:1163-1173. 
6. Finn RS, Zhu AX. Evolution of systemic therapy for hepatocellular carcinoma. Hepatology 2020 May 7. doi: 10.1002/hep.31306. [Epub ahead of print]

7. Kuzuya $T$, Ishigami $M$, Ito $T$, Ishizu $Y$, Honda $T$, Ishikawa $T$, et al. Sorafenib vs. lenvatinib as first-line therapy for advanced hepatocellular carcinoma with portal vein tumor thrombosis. Anticancer Res 2020;40:2283-2290.

8. Sung PS, Jang JW. Natural killer cell dysfunction in hepatocellular carcinoma: pathogenesis and clinical implications. Int J Mol Sci 2018;19:3648.

9. Keenan BP, Fong L, Kelley RK. Immunotherapy in hepatocellular carcinoma: the complex interface between inflammation, fibrosis, and the immune response. J Immunother Cancer 2019;7:267.

10. Yao W, Ba Q, Li X, Li H, Zhang $S$, Yuan $Y$, et al. A natural CCR2 antagonist relieves tumor-associated macrophage-mediated immunosuppression to produce a therapeutic effect for liver cancer. EBioMedicine 2017;22:58-67.

11. Ding W, Tan Y, Qian Y, Xue W, Wang Y, Jiang P, et al. Clinicopathologic and prognostic significance of tumor-associated macrophages in patients with hepatocellular carcinoma: a meta-analysis. PLoS One 2019;14:e0223971.

12. Finn RS, Ryoo BY, Merle P, Kudo M, Bouattour M, Lim HY, et al. Pembrolizumab as second-line therapy in patients with advanced hepatocellular carcinoma in KEYNOTE-240: a randomized, doubleblind, phase III trial. J Clin Oncol 2020;38:193-202.

13. Zhu AX, Finn RS, Edeline J, Cattan S, Ogasawara S, Palmer D, et al. Pembrolizumab in patients with advanced hepatocellular carcinoma previously treated with sorafenib (KEYNOTE-224): a nonrandomised, open-label phase 2 trial. Lancet Oncol 2018;19:940952.

14. El-Khoueiry AB, Sangro B, Yau T, Crocenzi TS, Kudo M, Hsu C, et al. Nivolumab in patients with advanced hepatocellular carcinoma (CheckMate 040): an open-label, non-comparative, phase 1/2 dose escalation and expansion trial. Lancet 2017;389:2492-2502.

15. Xu W, Liu K, Chen M, Sun JY, McCaughan GW, Lu XJ, et al. Immunotherapy for hepatocellular carcinoma: recent advances and future perspectives. Ther Adv Med Oncol 2019;11:1758835919862692.

16. Hilmi M, Neuzillet C, Calderaro J, Lafdil F, Pawlotsky JM, Rousseau B. Angiogenesis and immune checkpoint inhibitors as therapies for hepatocellular carcinoma: current knowledge and future research directions. J Immunother Cancer 2019;7:333.

17. Kimura T, Kato Y, Ozawa Y, Kodama K, Ito J, Ichikawa K, et al. Immunomodulatory activity of lenvatinib contributes to antitumor activity in the Hepa1-6 hepatocellular carcinoma model. Cancer Sci 2018;109:3993-4002.

18. Kim TH, Kim SY, Tang A, Lee JM. Comparison of international guidelines for noninvasive diagnosis of hepatocellular carcinoma: 2018 update. Clin Mol Hepatol 2019;25:245-263.

19. European Association for the Study of the Liver, European Association for the Study of the Liver. EASL Clinical Practice Guidelines: management of hepatocellular carcinoma. J Hepatol 2018;69:182236.

20. Llovet JM, Lencioni R. mRECIST for HCC: performance and novel refinements. J Hepatol 2020;72:288-306.

21. Park DJ, Sung PS, Kim JH, Lee GW, Jang JW, Jung ES, et al. EpCAMhigh liver cancer stem cells resist natural killer cell-mediated cytotoxicity by upregulating CEACAM1. J Immunother Cancer 2020;8:e000301.

22. Kato Y, Tabata K, Kimura T, Yachie-Kinoshita A, Ozawa Y, Yamada $K$, et al. Lenvatinib plus anti-PD-1 antibody combination treatment activates CD8 + T cells through reduction of tumor-associated macrophage and activation of the interferon pathway. PLoS One 2019;14:e0212513.

23. Voron T, Colussi O, Marcheteau E, Pernot S, Nizard M, Pointet AL, et al. VEGF-A modulates expression of inhibitory checkpoints on CD8+ T cells in tumors. J Exp Med 2015;212:139-148.

24. Kim CG, Jang M, Kim Y, Leem G, Kim KH, Lee H, et al. VEGF-A drives TOX-dependent $\mathrm{T}$ cell exhaustion in anti-PD-1-resistant microsatellite stable colorectal cancers. Sci Immunol 2019;4:eaay0555.

25. Zhou J, Tang Z, Gao S, Li C, Feng Y, Zhou X. Tumor-associated macrophages: recent insights and therapies. Front Oncol 2020;10:188.

26. Degroote $H$, Van Dierendonck A, Geerts A, Van Vlierberghe $H$, Devisscher $\mathrm{L}$. Preclinical and clinical therapeutic strategies affecting tumor-associated macrophages in hepatocellular carcinoma. J Immunol Res 2018;2018:7819520.

27. Singhal S, Stadanlick J, Annunziata MJ, Rao AS, Bhojnagarwala PS, O'Brien $S$, et al. Human tumor-associated monocytes/macrophages and their regulation of $T$ cell responses in early-stage lung cancer. Sci Transl Med 2019;11:eaat1500. 